\title{
The $S_{5}$ Extensions of Degree 6 with Minimum Discriminant
}

\author{
David Ford, Michael Pohst, Mario Daberkow, and Nasser Haddad
}

\section{CONTENTS}

\section{Introduction}

2. Distinguishing Galois Group $\mathrm{S}_{5}$

3. Signature $(6,0)$

4. Signatures $(2,2)$ and $(0,3)$

Table of Primitive Sextic Fields of Minimal Discriminant

Bibliography

Research supported by the Natural Sciences and Engineering Research Council (Canada) and Fonds pour la Formation de Chercheurs et l'Aide à la Recherche (Québec).

\begin{abstract}
The algebraic number fields of degree 6 having Galois group $\mathrm{S}_{5}$ and minimum discriminant are determined for signatures $(0,3)$, $(2,2)$ and $(6,0)$. The fields $F_{0}, F_{2}, F_{6}$ are generated by roots of $f_{0}(t)=t^{6}+3 t^{4}+2 t^{3}+6 t^{2}+1, f_{2}(t)=t^{6}-2 t^{4}+12 t^{3}-16 t+8$, and $f_{6}(t)=t^{6}-18 t^{4}+9 t^{3}+90 t^{2}-70 t-69$ respectively. Each of these fields is unique up to isomorphism. This completes the enumeration of primitive sextic fields with minimum discriminant for all possible combinations of Galois group and signature.
\end{abstract}

\section{INTRODUCTION}

The primitive algebraic number fields of a given degree having discriminant within given bounds may be enumerated by the method of [Pohst 1982]. This approach is applied in [Pohst et al. 1982] to determine the sextic fields of minimum discriminant with Galois group $S_{6}$ (signatures $(6,0),(4,1)$ and $(0,3))$ and in [Olivier 1990] for Galois groups $S_{6}$ (all signatures) and $A_{5} \simeq \mathrm{PSL}_{2}\left(\mathbb{F}_{5}\right)$ and $A_{6}$ (signature $(2,2)$ only). As we will show, the method suffices as well to determine the fields of minimum discriminant for the group $S_{5} \simeq \mathrm{PGL}_{2}\left(\mathbb{F}_{5}\right)$, except in the totally real case.

The method is not adequate for investigating primitive totally real sextic fields; too many examples are generated. A refined method was developed to reduce the examples to a manageable number, and the totally real sextic fields of minimum discriminant with Galois groups $A_{5}$ [Ford and Pohst 1992] and $A_{6}$ [Ford and Pohst 1993] were determined.

The number of examples produced by this improved method is still enormous. In searching for 
fields with alternating Galois groups $\left(A_{5}\right.$ and $\left.A_{6}\right)$ it is effective to screen out polynomials with nonsquare discriminant. For the group $S_{5}$ this technique is not available. In its place we use a more costly screening method based on mod- $p$ polynomial factorization, the efficiency of which is critical for the feasibility of the computation.

\section{DISTINGUISHING GALOIS GROUP $\mathrm{S}_{5}$}

The group $\mathrm{PGL}_{2}\left(\mathbb{F}_{5}\right)$ is generated as a permutation group by (1 23245$)$ and (1 6)(2 3)(4 5); the cycle types $1 \cdot 1 \cdot 1 \cdot 1 \cdot 2,1 \cdot 1 \cdot 1 \cdot 3,1 \cdot 2 \cdot 3$ and $2 \cdot 4$ do not occur in $\mathrm{PGL}_{2}\left(\mathbb{F}_{5}\right)$. So if $f$ is a polynomial with Galois group $\mathrm{PGL}_{2}\left(\mathbb{F}_{5}\right)$ and $p$ is a prime not dividing the discriminant of $f$ then the degree sequence of the $\bmod p$ factors of $f$ cannot be among these four types [van der Waerden 1966, Section 8.10].

We generate polynomials of the form

$f(t)=t^{6}+a_{1} t^{5}+a_{2} t^{4}+a_{3} t^{3}+a_{4} t^{2}+a_{5} t+a_{6} \in \mathbb{Z}[t]$,

the coefficients being determined in the order $a_{1}$, $a_{2}, a_{6}, a_{3}, a_{4}, a_{5}$.

For each triple $\left(a_{1}, a_{2}, a_{6}\right)$ and for each $p$ in a suitably chosen set of primes $\left\{p_{1}, \ldots, p_{n}\right\}$ we define a flag $I_{p}$ and Boolean arrays $V_{p}$ and $W_{p}$.

\begin{tabular}{ccl} 
NAME & SPACE & \multicolumn{1}{c}{ DEFINITION } \\
$I_{p}$ & $n$ & $\begin{array}{l}\text { Has } V_{p} \text { been initialized } \\
\text { (to False) } ?\end{array}$ \\
$V_{p}\left[r_{3}, r_{4}, r_{5}\right]$ & $\sum p^{3}$ & $\begin{array}{l}\text { Has } W_{p}\left[r_{3}, r_{4}, r_{5}\right] \\
\text { been computed? }\end{array}$ \\
$W_{p}\left[r_{3}, r_{4}, r_{5}\right]$ & $\sum p^{3}$ & $\begin{array}{l}\text { Does } t^{6}+a_{1} t^{5}+a_{2} t^{4}+r_{3} t^{3}+ \\
r_{4} t^{2}+r_{5} t+a_{6} \text { give a cycle } \\
\end{array}$ \\
& & type from $\mathrm{PGL}_{2}\left(\mathbb{F}_{5}\right) ?$
\end{tabular}

The values $r_{3}, r_{4}, r_{5}$ are the residues of $a_{3}, a_{4}, a_{5}$ $\bmod p$. When $p$ divides the discriminant of $t^{6}+$ $a_{1} t^{5}+a_{2} t^{4}+r_{3} t^{3}+r_{4} t^{2}+r_{5} t+a_{6}$, its $\bmod p$ factorization gives no information, so we regard $W_{p}\left[r_{3}, r_{4}, r_{5}\right]$ as True.

The polynomial $f(t)$ is excluded if $W_{p}\left[r_{3}, r_{4}, r_{5}\right]$ is False for some $p$ in $\left\{p_{1}, \ldots, p_{n}\right\}$.
When $\left|a_{6}\right|$ is large few triples $\left(a_{3}, a_{4}, a_{5}\right)$ are generated. In such cases it is usual that these few polynomials are all excluded using only a few small primes, and it is worthwhile to avoid taking time to initialize $V_{p}$ for the larger values of $p$.

When $\left|a_{6}\right|$ is small, many triples $\left(a_{3}, a_{4}, a_{5}\right)$ are generated and all the primes are used. For these cases it is advantageous to have the number of primes as large as possible.

\section{SIGNATURE $(6,0)$}

We are to generate at least one defining polynomial $f(t)$ of the form (2.1) for each primitive sextic algebraic number field $F$ with signature $(6,0)$ and discriminant $d_{F} \leq B=767431973$. Taking $a_{1} \in\{0,1,2,3\}$ and $\rho$ a root of $f$ we have

$$
\operatorname{Tr}\left(\rho^{2}\right) \leq \frac{1}{6} a_{1}^{2}+\left(\frac{4}{3} B\right)^{1 / 5}
$$

by [Cohen 1993, Theorem 6.4.2], which for successive values of $a_{1}$ gives bounds of $63.386,63.553$, 64.053 and 64.886 for $\operatorname{Tr}\left(\rho^{2}\right)$. Because $F$ is totally real we have

$$
\operatorname{Tr}\left(\rho^{2}\right)=a_{1}^{2}-2 a_{2} \equiv a_{1} \bmod 2,
$$

so that $\operatorname{Tr}\left(\rho^{2}\right)$ is bounded by $62,63,64,63$ for $a_{1}=$ $0,1,2,3$ respectively. Bounds on the coefficients $a_{2}, a_{6}, a_{3}, a_{4}, a_{5}$ are determined as in [Ford and Pohst 1992].

The polynomials are screened for cycle-type compatibility with $\mathrm{PGL}_{2}\left(\mathbb{F}_{5}\right)$ using the technique of section 2 with the twenty-five primes in the range $2 \leq p \leq 97$.

The computation required about 13720 CPUhours on a Digital VAXstation 4000-90 in the Computer Science Department at Concordia University (the same system used in [Ford and Pohst 1993]). The cases with $\operatorname{Tr}\left(\rho^{2}\right) \geq 55$ were independently confirmed on a network of thirty UNIX workstations at the Technische Universität Berlin.

Theorem 3.1. The minimum possible discriminant for a totally real $S_{5}$ extension of degree 6 is $d_{6}=$ $767431973=7^{3} 11^{3} 41^{2}$. There is, up to isomorphy, 
exactly one field $F_{6}$ of that discriminant with $\mathrm{Ga}$ lois group $S_{5}$. It is generated by a root $\rho_{6}$ of the polynomial

$$
f_{6}(t)=t^{6}-18 t^{4}+9 t^{3}+90 t^{2}-70 t-69 .
$$

The class number of $F_{6}$ is 1 . An integral basis for $F_{6}$ is given by

$$
1, \quad \rho_{6}, \quad \rho_{6}^{2}, \quad \rho_{6}^{3}, \quad \rho_{6}^{4}, \quad \rho_{6}^{5} .
$$

A system of fundamental units for $F_{6}$ is

$$
\begin{aligned}
& \varepsilon_{61}=2-\rho_{6}, \\
& \varepsilon_{62}=8+8 \rho_{6}-10 \rho_{6}^{2}+\rho_{6}^{4}, \\
& \varepsilon_{63}=71+102 \rho_{6}-47 \rho_{6}^{2}-27 \rho_{6}^{3}+5 \rho_{6}^{4}+2 \rho_{6}^{5}, \\
& \varepsilon_{64}=104+90 \rho_{6}-50 \rho_{6}^{2}-26 \rho_{6}^{3}+5 \rho_{6}^{4}+2 \rho_{6}^{5}, \\
& \varepsilon_{65}=101+129 \rho_{6}-63 \rho_{6}^{2}-38 \rho_{6}^{3}+7 \rho_{6}^{4}+3 \rho_{6}^{5} .
\end{aligned}
$$

\section{SIGNATURES $(2,2)$ AND $(0,3)$}

We generate at least one defining polynomial $f(t)$ for each primitive sextic algebraic number field $F$ with $\left|d_{F}\right| \leq B=2299968$ as in section 3 of [Pohst 1982], with slight variations. Taking $\rho^{(1)}, \ldots, \rho^{(6)}$ to be the algebraic conjugates of a root $\rho$ of $f(t)$ and $m>0$, we define

$$
S_{m}(\rho)=\sum_{j=1}^{6}\left(\rho^{(j)}\right)^{m} \quad \text { and } \quad T_{m}(\rho)=\sum_{j=1}^{6}\left|\rho^{(j)}\right|^{m} .
$$

For $a_{1}=0,1,2,3$ the respective bounds on $T_{2}(\rho)$ given by [Cohen 1993, Theorem 6.4.2] are 19.830, 19.997, 20.497 and 21.330. Bounds on $\left\lfloor T_{3}(\rho)\right\rfloor$, $\left\lfloor T_{4}(\rho)\right\rfloor,\left\lfloor T_{5}(\rho)\right\rfloor$ and $\left\lfloor T_{6}(\rho)\right\rfloor$ follow according to [Pohst 1982, Theorem 4], and bounds for $a_{2}, a_{6}, a_{3}$, $a_{4}, a_{5}$ are then determined in the usual way, using the facts that $S_{m}(\rho) \in \mathbb{Z}$ and $\left|S_{m}(\rho)\right| \leq\left\lfloor T_{m}(\rho)\right\rfloor$.

The polynomials are screened for cycle-type compatibility with $\mathrm{PGL}_{2}\left(\mathbb{F}_{5}\right)$ and tested for irreducibility. Due to system restrictions the screening technique of section 2 was applied only for the twenty primes in the range $2 \leq p \leq 71$.
The computation required about 441 CPU-hours on a Digital AlphaServer 2100 4/200 in the Department of Computing Services at Concordia University (for polynomial generation and screening), plus a small amount of time on other systems (for computing signatures, field discriminants, Galois groups, class groups and fundamental units).

Theorem 4.1. The minimum discriminant for an $S_{5}$ extension of degree 6 and signature $(2,2)$ is $d_{2}=$ $2299968=2^{6} 3^{3} 11^{3}$. There is, up to isomorphy, exactly one field $F_{2}$ of that discriminant with $G a-$ lois group $S_{5}$. It is generated by a root $\rho_{2}$ of the polynomial

$$
f_{2}(t)=t^{6}-2 t^{4}+12 t^{3}-16 t+8 .
$$

The class number of $F_{2}$ is 1 . An integral basis for $F_{2}$ is given by

$$
1, \quad \rho_{2}, \quad \omega_{2}=\frac{1}{2} \rho_{2}^{2}, \quad \rho_{2} \omega_{2}, \quad \omega_{2}^{2}, \quad \rho_{2} \omega_{2}^{2} .
$$

A system of fundamental units for $F_{2}$ is

$$
\begin{aligned}
& \varepsilon_{21}=\omega_{2}, \\
& \varepsilon_{22}=1-3 \rho_{2}+6 \omega_{2}-\omega_{2}^{2}+\rho_{2} \omega_{2}, \\
& \varepsilon_{23}=-3+3 \rho_{2}+5 \omega_{2}-\rho_{2} \omega_{2}+\omega_{2}^{2}+\rho_{2} \omega_{2}^{2} .
\end{aligned}
$$

Theorem 4.2. The discriminant of minimum absolute value for a totally complex $S_{5}$ extension of degree 6 is $d_{0}=-1778112=-2^{6} 3^{4} 7^{3}$. There is, up to isomorphy, exactly one field $F_{0}$ of that discriminant with Galois group $S_{5}$. It is generated by a root $\rho_{0}$ of the polynomial

$$
f_{0}(t)=t^{6}+3 t^{4}+2 t^{3}+6 t^{2}+1 .
$$

The class number of $F_{0}$ is 1 . An integral basis for $F_{0}$ is given by

$$
\begin{aligned}
1, \quad \rho_{0}, \quad \rho_{0}^{2}, \quad \rho_{0}^{3}, \quad \rho_{0}^{4}, \\
\omega_{0}=\frac{1}{3}\left(1-\rho_{0}+\rho_{0}^{2}+\rho_{0}^{3}-\rho_{0}^{4}+\rho_{0}^{5}\right) .
\end{aligned}
$$

$A$ system of fundamental units for $F_{0}$ is

$$
\varepsilon_{01}=\rho_{0}, \quad \varepsilon_{02}=-1+\rho_{0}+\rho_{0}^{2}+\rho_{0}^{3} .
$$

This result is reported in [Haddad 1996]. 


$\begin{array}{ccrll}\text { Group } & \text { Signature } & \text { DisCRim. } & \text { GENERATING POlYNOMIAL } & \text { REFERENCE } \\ A_{5} & (6,0) & 30991489 & t^{6}-10 t^{4}+7 t^{3}+15 t^{2}-14 t+3 & \text { [Ford and Pohst 1992] } \\ & (2,2) & 287296 & t^{6}+2 t^{5}+t^{4}+4 t^{3}+2 t^{2}-4 t+1 & \text { [Olivier 1990] } \\ A_{6} & (6,0) & 170067681 & t^{6}-24 t^{4}+21 t^{2}+9 t+1 & \text { [Ford and Pohst 1993] } \\ & (2,2) & 287296 & t^{6}+2 t^{5}-t^{4}+2 t^{2}-1 & \text { [Olivier 1990] } \\ S_{5} & (6,0) & 767431973 & t^{6}-18 t^{4}+9 t^{3}+90 t^{2}-70 t-69 & - \\ & (2,2) & 2299968 & t^{6}-2 t^{4}+12 t^{3}-16 t+8 & - \\ & (0,3) & -1778112 & t^{6}+3 t^{4}+2 t^{3}+6 t^{2}+1 & - \\ S_{6} & (6,0) & 592661 & t^{6}-5 t^{5}+2 t^{4}+18 t^{3}-11 t^{2}-19 t+1 & \text { [Pohst et al. 1982] } \\ & (4,1) & -92779 & t^{6}+t^{5}-2 t^{4}-3 t^{3}-t^{2}+2 t+1 & \text { [Pohst et al. 1982] } \\ & (2,2) & 29077 & t^{6}+2 t^{5}-t^{4}-t^{2}-t+1 & \text { [Olivier 1990] } \\ & (0,3) & -14731 & t^{6}+t^{5}-t^{3}-t^{2}+1 & \text { [Pohst et al. 1982] }\end{array}$

Primitive sextic fields of minimal discriminant.

\section{REFERENCES}

[Cohen 1993] H. Cohen, A course in computational algebraic number theory, Graduate Texts in Mathematics 138, Springer, Berlin, 1993.

[Ford and Pohst 1992] D. Ford and M. Pohst, "The totally real $A_{5}$ extension of degree 6 with minimum discriminant", Experiment. Math. 1:3 (1992), 231235.

[Ford and Pohst 1993] D. Ford and M. Pohst, "The totally real $A_{6}$ extension of degree 6 with minimum discriminant", Experiment. Math. 2:3 (1993), 231232.

[Haddad 1996] N. Haddad, The totally complex sextic $S_{5}$ extension of minimum discriminant, M.Sc. thesis, Concordia University, 1996.
[Olivier 1990] M. Olivier, "Corps sextiques primitifs", Ann. Inst. Fourier (Grenoble) 40:4 (1990), 757-767.

[Pohst 1982] M. Pohst, "On the computation of number fields of small discriminants including the minimum discriminants of sixth degree fields", J. Number Theory 14:1 (1982), 99-117.

[Pohst et al. 1982] M. Pohst, P. Weiler, and H. Zassenhaus, "On effective computation of fundamental units, II", Math. Comp. 38:157 (1982), 293-329.

[van der Waerden 1966] B. L. van der Waerden, Algebra, 7th ed., Grundlehren der Mathematischen Wissenschaften 33, Springer, Berlin, 1966. Translated as Algebra, Frederick Ungar Publishing Co., New York, 1970 (reprinted by Springer, New York, 1991).

David Ford, Department of Computer Science, Concordia University, 1455 de Maisonneuve Blvd. West, Montreal, Quebec H3G 1M8, Canada (ford@cicma.concordia.ca)

Michael Pohst, Fachbereich 3 Mathematik MA 8-1, Technische Universität Berlin, Straße des 17. Juni 136, 10623 Berlin, Germany (pohst@math.tu-berlin.de)

Mario Daberkow, Fachbereich 3 Mathematik, Technische Universität Berlin, Straße des 17. Juni 136, 10623 Berlin, Germany (daberkow@math.berkeley.edu)

Nasser Haddad, Department of Mathematics, Concordia University, 1455 de Maisonneuve Blvd. West, Montreal, Quebec H3G 1M8, Canada (haddad@cicma.concordia.ca) 


\section{Instructions for Authors}

Experimental Mathematics is devoted to experimental aspects of mathematical research. It publishes proved results inspired by experimentation, conjectures suggested by experiments, surveys of certain areas from the experimental point of view, descriptions of algorithms and software for mathematical exploration, and general articles of interest to the community. A more detailed statement of philosophy and of the publishability criteria is available on the World-Wide Web at http://www . expmath. org, or by request from the publisher (see address below, or send e-mail to expmath@ expmath.org).

\section{Submission Information}

To submit a contribution, send four copies of the material, together with a cover letter stating that it is intended for publication in Experimental Mathematics, to:

Experimental Mathematics

A K Peters, Ltd.

289 Linden Street

Wellesley, MA 02181

phone: $617-235-2210$

You are encouraged to send your text in electronic form as well; see the section on Electronic Text at the end of this document.

Submission of a paper implies that the work has not been published before, except perhaps in the form of an abstract or as part of a lecture, review, or thesis; that it is not under consideration for publication elsewhere; that its publication has been approved by all authors and (if appropriate) by the institution where the work was carried out; that, if and when the manuscript is accepted for publication, the authors agree to automatic transfer of the copyright to the publisher; and that the manuscript will not be published elsewhere in any language with the consent of the copyright holders. Submissions will be acknowledged, but not be returned.

\section{Charges}

There are no page charges for publications, but authors are expected to contribute toward the cost of color illustrations in their articles. Rates will take into account funding available to authors and editorial necessity.

\section{Offprints}

Authors will receive in all 25 free offprints of their work. At production time authors may order up to 75 additional offprints at cost.

\section{Manuscript Requirements}

Manuscripts must be in English, French or German. They should be written clearly and concisely. We reserve the right to edit contributions for style and format, with changes subject to the authors' approval.

All submissions must include the following elements:

1. title and (if title exceeds 75 characters) alternative short title for running heads;

2. postal address, affiliation (if appropriate) and electronic address (if available) for each author;

3. an abstract of at most 150 words, in the same language as the article, and an English translation if the article is not in English.

\section{References}

References should include full information: author or institution; full title; publisher, city and year (for books, manuals, etc.), or full journal name, volume, year and page range (for papers). References to software should contain complete manufacturer's or distributor's names and addresses. All references in the bibliography should be cited in the text, or accompanied by comments stating their relevance. Reference tags in the text should include author's last name and year of publication, in brackets [Poincaré 1901]. Use a comma to separate a tag from a subsequent page or section number, and semicolons to separate several tags in the same brackets.

\section{Figures}

The following types of figures are acceptable:

1. traditional hand-drawn figures, in india ink on glossy paper or vellum;

2. black-and-white and color photographs, of reproduction quality, mounted on $8 \frac{1}{2} \times 11$-inch cardboard;

3. electronically generated figures. 
For hand-drawn figures and photographs, an original and three clear copies should accompany the four copies of the text.

For electronically generated figures, you can use photographs or printouts for the submission, but you must supply the electronic source files if your article is accepted for publication. Under no circumstances will we reproduce low-resolution hard copy or screen photographs.

Figure source files should be in Encapsulated PostScript (EPS) or in a form that can be converted to EPS, such as GnuPlot or Mathematica input. Many drawing tools such as Adobe Illustrator and Aldus FreeHand can produce EPS output. If your figure contains bitmaps, please generate them at the highest possible resolution: before taking a screen dump, for example, resize the window, if possible, to occupy the whole screen. When in doubt whether your figure source is in an acceptable format, check with the editors by sending electronic mail to expmath@expmath.org.

For each figure, please supply a caption and a number by which the figure is referred to in the text. If possible, integrate the figures with the text; otherwise, indicate their optimal placement by means of a comment such as "Place Figure 1 here". In referring to the figure, avoid constructions ("the curve looks like this:") that require the exact placement to be known in advance.

See also the section on Charges, above.

\section{Programs}

Experimental Mathematics does not publish programs in printed form. You can include short illustrative excerpts from your programs, either within the text itself (if at most three lines) or as a separate display. Please supply a caption and a number for each displayed listing. Keep in mind that many readers will not be familiar with the programming language in which your program is written; it is almost always better to explain what a program does in words than to let the program speak for itself.

Similar considerations apply to program output and interactive sessions.

\section{Electronic Text}

If your article is accepted, it is helpful for us to have the text in electronic form. You can transfer it by e-mail, ftp, or diskette. Send e-mail to expmath@expmath.org for details.

Experimental Mathematics is typeset in $\mathrm{LAT}_{\mathrm{EX}}$. This means that the production time is shorter if the article is written in $\mathrm{LT}_{\mathrm{E} X} \mathrm{X}$ or other variants of $\mathrm{T}_{\mathrm{E}} \mathrm{X}$ than otherwise. However, having the text in electronic form helps even if it is not in $\mathrm{T}_{\mathrm{EX}}$.

Most word-processing and typesetting systems allow you to save the copy in text-only or ASCII mode, where the formatting codes are discarded, and only the text is kept, in a format that approximates that of the typeset document as well as possible. Please use this option when saving your text for production in Experimental Mathematics.

The rest of this section concerns authors who are using $\mathrm{T}_{\mathrm{E}} \mathrm{X}$ in one of its variants. Here again there are many things you can do to help the editor's and compositor's work and expedite production.

You should preferably use IATEX's article style or $\mathcal{A}_{\mathcal{M}} \mathcal{S}$-TEX's amsppt style. Whether you use one of these styles, another style, or another variant of $T_{E X}$ such as plain $\mathrm{T}_{\mathrm{E}} \mathrm{X}$, indicate at the top of the file what system you're using.

Don't use two-column format.

Be as consistent as possible in using your own macros. Put them into a file that is input at the top the document, after all style files. Do not embed any new definitions in your text. Avoid redefining existing $\mathrm{T}_{\mathrm{E} X}$, $\mathcal{A} \mathcal{M}$ - $\mathrm{T}_{\mathrm{EX}}$ or $\mathrm{IAT}_{\mathrm{E}} \mathrm{X}$ commands.

Avoid using explicit vertical spacing commands such as \vskip, \medskip, \bigbreak. Default spacing is provided by \beginsection, \proclaim, \demo, etc. in $\mathcal{A} \mathcal{M}$ - $\mathrm{TE}_{\mathrm{E}} \mathrm{X}$ and plain $\mathrm{TEX}_{\mathrm{E}}$, and by $\backslash$ begin $\{$ theorem\}, Isection, etc., in IATEX. To set off a paragraph or a portion of your text other than proofs, theorems, exercises, etc., you may add extra space, but please provide the compositor with a comment line (a line preceded by $\%$ ) to make sure this space will not be eliminated in reformatting.

Likewise, avoid using explicit horizontal spacing commands. If you must use extra spacing, do it consistently, by means of a macro that can be adjusted globally by the compositor if necessary. Please add a comment if a specific spacing convention is to be retained.

Do not, under any circumstances, insert forced line breaks or page breaks in your document. There is no point in your trying to optimize line breaks and page breaks in the original manuscript, since they will not be preserved in the journal's two-column format. Forced breaks just confuse the compositor.

Set off displayed equations with $\$ \$$ on a line by itself.

In general, if you want certain elements to be kept together, or displayed in a particular fashion, add a comment line for the compositor in your electronic files or indicate it in your hard copy. 